\title{
Improved Magnetic Fusion Energy Economics Via Massive Resistive Electromagnets
}

\author{
Robert D. Woolley \\ Princeton University \\ Princeton Plasma Physics Laboratory* \\ P.O. Box 451 \\ Princeton, New Jersey 08543 \\ (609) 243-3130 \\ *Supported by U.S.Department of Energy Contract No. DE-AC02-76CH03073.
}

\section{ABSTRACT}

Abandoning superconductors for magnetic fusion reactors and instead using resistive magnet designs based on cheap copper or aluminum conductor material operating at "room temperature" $\left(300^{\circ} \mathrm{K}\right)$ can reduce the capital cost per unit fusion power and simplify plant operations. ${ }^{1}$ By increasing unit size well beyond that of present MFE conceptual designs using superconducting electromagnets, the recirculating power fraction needed to operate resistive electromagnets can be made as close to zero as needed for economy without requiring superconductors. Other advantages of large fusion plant size, such as very long inductively driven pulses, may also help reduce the cost per unit fusion power. ${ }^{2}$

\section{INTRODUCTION}

Although the deuterium and lithium raw materials for DT fusion fuels are cheaper, cleaner, and more plentiful than fossil fuels (i.e., less than $\$ 0.001$ per thermal Gigajoule for fusion versus $\$ 2.32$ for natural gas), the conventional vision for fusion is more expensive. Today's conceptual designs of future commercial MFE (magnetic fusion energy) power plants provide two reasons for this situation:

-very high capital cost per watt of output power

-very high maintenance cost

To put the capital cost issue into perspective, consider the following comparison, which assumes the additional cost of MFE Powerplant systems not present in the ITER experiment will be offset by future cost reduction progress:

Table 1: Power Production Capital Costs

\begin{tabular}{|l|l|l|l|}
\hline Technology & Output Power & CapitalCost & $\begin{array}{l}\text { Capital } \\
\text { Cost/watt }\end{array}$ \\
\hline AutomobileEngine & $\begin{array}{c}100 \mathrm{~kW} \\
(134 \mathrm{hp})\end{array}$ & $\$ 2000$ & $\$ 0.02$ \\
\hline $\begin{array}{l}\text { GasTurbine/Elect. } \\
\text { Gen }\end{array}$ & $160 \mathrm{MW}$ & $\$ 40 \mathrm{M}$ & $\$ 0.25$ \\
\hline $\begin{array}{l}\text { Superconducting } \\
\text { MFE Powerplant }\end{array}$ & $0.5 \mathrm{GW}(\mathrm{el})$ & $\$ 10 \mathrm{~B}$ & $\$ 20.00$ \\
\hline
\end{tabular}

In MFE power plant designs, the reliance on superconducting electromagnets is a big contributor to high capital cost. Today's liquid helium temperature superconductors incorporate niobium, a rare and expensive material compared to copper or aluminum. In addition to the direct costs for constructing the large superconducting electromagnets, there are also direct costs for cryogenic refrigeration plants needed to maintain the magnets' temperature near absolute zero, direct costs for electric energy dump systems needed to protect the superconducting magnets from internal damage when "quench" events occur, and indirect costs for the additional unproductive magnetized volume housing the superconducting magnets' necessary radiation shielding and thermal insulation. There are also large operating costs associated with the (very long) lost time periods required for transient cooldown and heatup of the superconductors.

Even at the smallest physical size now considered for MFE power plants, each toroidal field (TF) electromagnet is too large to transport in one piece on roads. Large size practically requires a demountable design in which the electromagnets are composed of smaller modules with demountable electrical joints, individually manufactured, transported, and assembled at the plant site. Demountable magnets for fusion experiments have frequently been built using resistive conductors, but are difficult with cryogenic superconductors and have never been engineered. Since there are no viable demountable superconducting electromagnet designs it is therefore necessary to manufacture each superconducting electromagnet locally, at each MFE plant site. This impediment to efficient centralized manufacturing contributes to the high projected cost of superconducting MFE power plants.

The lack of demountable TF superconducting magnet designs also has other impacts. The initial assembly of the MFE power plant is difficult, time consuming, and expensive, requiring that vacuum vessel segments be "threaded" through intact TF coils. And if a superconducting TF coil were to fail after the vacuum vessel has become contaminated by DT operation, economic replacement of the TF coil seems impossible.

\section{FUSION POWER SCALING vs. MAGNET SCALING}

Small high field water cooled electromagnets do not appear attractive for fusion applications. Heat removal dominates their design and they seem to waste a lot of electricity. The accelerator community has largely abandoned water cooled electromagnets in favor of superconductors. How could the fusion community consider going in the opposite direction? The key point allowing resistive magnets to be used for fusion power applications is that the required electromagnet current density decreases as the designed size of the electromagnet is increased. Fusion electromagnets are typically $>10$ times the linear size of accelerator electromagnets, so their current densities are reduced by a 
factor $>10$. Their power densities are thus reduced by a factor $>100$, so their cooling becomes relatively easy.

The total "threading" current (in ampere-turns) required in the toroidal field (TF) electromagnet of a tokamak is given by

$$
\mathrm{I}=\left(2 \pi / \mu_{0}\right) \mathrm{B} R
$$

If the geometric shape of a $\mathrm{TF}$ electromagnet is kept constant while its size is varied, the conductor cross section area varies as $\mathrm{R}^{2}$, so TF conductor current density scales as

$$
\mathrm{J} \propto \mathrm{B} / \mathrm{R}
$$

In a resistive magnet with constant resistivity $\eta$, the local dissipated power density in the conductor is

$$
P_{\text {density }}=\eta J^{2} \propto B^{2} R^{-2}
$$

With the total conductor volume varying with size proportional to $\mathrm{R}^{3}$, it follows that total dissipated magnet power varies as

$$
\mathrm{P}_{\text {magnet }} \propto \mathrm{B}^{2} \mathrm{R}^{1}
$$

So for resistive MFE power plant designs, electromagnet power at constant field increases only as the cube root of the plasma volume. On the other hand, fusion power at constant field and plasma properties is proportional to plasma volume, not to its cube root. If the geometrical shape, temperature, and composition of an ignited plasma is kept constant while its overall pressure, magnetic field, and size are varied, total fusion power varies as

$$
\mathrm{P}_{\text {fusion }} \propto \beta^{2} \mathrm{~B}^{4} \mathrm{R}^{3}
$$

where linear size variation is represented by the plasma major radius, $\mathrm{R}$, and where $\beta$ is the plasma pressure normalized to the magnetic field pressure.

In a DT fusion power plant, the total thermal power produced is the sum of DT fusion power from the basic DT fusion reaction,

$$
{ }_{1} \mathrm{D}^{2}+{ }_{1} \mathrm{~T}^{3} \rightarrow{ }_{2} \mathrm{He}^{4}+{ }_{0} \mathrm{n}^{1}+17.6 \mathrm{Mev},
$$

and the power produced by additional nuclear reactions occurring in the blanket such as the exothermic reaction producing tritium from $\mathrm{Li}^{6}$,

$$
{ }_{3} \mathrm{Li}^{6}+{ }_{0} \mathrm{n}^{1} \rightarrow{ }_{1} \mathrm{~T}^{3}+{ }_{2} \mathrm{He}^{4}+4.8 \mathrm{Mev} .{ }^{3}
$$

Total thermal power can be represented as

$$
\mathrm{P}_{\text {thermal }}=\mathrm{M} \mathrm{P}_{\text {fusion }}
$$

In an ideal thick blanket composed only of ${ }_{3} \mathrm{Li}^{6}$ (no structure, no neutron multiplier), the blanket energy multiplier factor, $\mathrm{M}$, would be

$$
\mathrm{M}=(17.6 \mathrm{Mev}+4.8 \mathrm{Mev}) /(17.6 \mathrm{Mev})=1.27
$$

while the tritium breeding ratio (TBR) which is defined as the number of tritium atoms produced per tritium atom consumed would be 1.00. Natural lithium contains the isotope, ${ }_{3} \mathrm{Li}^{7}$ in addition to ${ }_{3} \mathrm{Li}^{6}$, which allows the endothermic reaction

$$
{ }_{3} \mathrm{Li}^{7}+{ }_{0} \mathrm{n}^{1} \rightarrow \mathrm{T}^{3}+{ }_{2} \mathrm{He}^{4}+{ }_{0} \mathrm{n}^{1}-2.5 \mathrm{Mev}
$$

to also occur in the blanket for neutrons with energies above 2.5 Mev. Depending on geometry, a natural lithium blanket can increase its TBR well above 1.00 with a reduced value of $\mathrm{M}$. For examples, detailed numerical computations for some idealized natural lithium blankets have estimated $(\mathrm{M}, \mathrm{TBR})=(1.12,1.65)$ and $(1.19,1.89) .{ }^{4}$ However, a realistic natural lithium blanket would contain structure with neutron moderating properties which could reduce ${ }_{3} \mathrm{Li}^{7}$ reactions thus increasing $\mathrm{M}$ closer to 1.27 .

Gross electric power output represents thermal conversion at efficiency $\varepsilon$, assumed in the range, $0.33<\varepsilon<0.45$. Then

$$
\mathrm{P}_{\text {gross-elect }}=\varepsilon \mathrm{P}_{\text {thermal }}=\varepsilon \mathrm{M} \mathrm{P}_{\text {fusion }} \propto \mathrm{P}_{\text {fusion }}
$$

In a MFE power plant using superconducting electromagnets, a small portion of the gross electric power must be recirculated to operate the necessary cryogenic refrigerators. Similarly, in a MFE power plant using resistive electromagnets, a recirculating fraction, $f$, of the gross electric power output must be recycled to operate the electromagnets. The fraction, $\mathrm{f}$, is given by

$$
\begin{array}{r}
\mathrm{f}=\left(\mathrm{P}_{\text {magnet }} / \mathrm{P}_{\text {gross-elect }}\right) \\
\propto\left(\mathrm{B}^{2} \mathrm{R}^{1} / \beta^{2} \mathrm{~B}^{4} \mathrm{R}^{3}\right)=1 / \beta^{2} \mathrm{~B}^{2} \mathrm{R}^{2}
\end{array}
$$

Therefore, increasing $\beta, \mathrm{B}, \mathrm{R}$ all reduce the recirculating power fraction, $\mathrm{f}$, towards zero.

Since the net fusion power output is only (1-f) times the gross electrical output, the economic effect of recirculating power is to multiply the Cost Of Electricity (COE) for sale by the ratio, $1 /(1-f)$. It is clearly desirable that $f$ should be small, but with resistive magnets there is a tradeoff optimization between the cost of the magnets themselves and this COE multiplier.

The "wall load" power density is defined as power per unit wall area. It scales as

$\mathrm{P}_{\mathrm{Wall}}=\left(\mathrm{P}_{\text {fusion }} /\right.$ WallArea $) \propto \beta^{2} \mathrm{~B}^{4} \mathrm{R}^{1}$

Although the highest attainable wall load should be sought in order to effectively utilize first wall capital investment, 
the designed wall load must realistically be limited to a value which future first wall technology will be able to accommodate. The proper wall load design value is today somewhat uncertain. Although the ITER design develops about $1 \mathrm{MW} / \mathrm{m}^{2}$, some researchers consider the range from 5 to $10 \mathrm{MW} / \mathrm{m}^{2}$ as probably achievable, and wall loads even as high as $30 \mathrm{MW} / \mathrm{m}^{2}$ have been suggested as goals for exotic wall design concepts. Thus, $5 \mathrm{MW} / \mathrm{m}^{2}$ may be an achievable wall load.

Increasing either B or $\mathrm{R}$ by itself also increases the wall load. However, at a fixed wall load limit, $\mathrm{P}_{\text {wlim, we can }}$ vary them together while keeping the designed magnetic field a particular function of size and the plasma $\beta$, setting

$$
\mathrm{B} \propto \beta^{-1 / 2} \mathrm{R}^{-1 / 4} \mathrm{P}_{\text {Wlim }}{ }^{1 / 4}
$$

Then $\quad \mathrm{P}_{\text {wall }}=\mathrm{P}_{\text {wlim }}$

and the fusion and magnet power vary as

and

$$
\mathrm{P}_{\text {fusion }} \propto \mathrm{R}^{2} \mathrm{P}_{\text {wlim }}
$$

$$
\mathrm{P}_{\text {magnet }} \propto \beta^{-1} \mathrm{R}^{1 / 2} \mathrm{P}_{\text {wlim }}{ }^{1 / 2}
$$

The recirculating power fraction then varies as

$$
\mathrm{f} \propto\left(1 / \beta^{2} B^{2} R^{2}\right)=1 /\left(\beta^{1} R^{3 / 2} P_{\text {wlim }}{ }^{1 / 2}\right)
$$

Thus, increasing the size while reducing the magnetic field can reduce the recirculating power fraction needed to power resistive coils to as low a value as needed for economy, even while holding the wall load constant.

These relationships for operation at a fixed wall load limit can also be restated in terms of the gross ratings of the MFE power plant as

$$
\begin{aligned}
& \mathrm{f} \propto \beta^{-1} \mathrm{P}_{\text {fusion }}{ }^{-3 / 4} \mathrm{P}_{\text {wlim }}{ }^{1 / 4} \\
& \mathrm{P}_{\text {magnet }} \propto \beta^{-1} \mathrm{P}_{\text {fusion }}{ }^{1 / 4} \mathrm{P}_{\text {wlim }}{ }^{1 / 4} \\
& \mathrm{R} \propto \mathrm{P}_{\text {fusion }}{ }^{1 / 2} \mathrm{P}_{\text {Wlim }}{ }^{-1 / 2} \\
& \mathrm{~B} \propto \beta^{-1 / 2} \mathrm{P}_{\text {fusion }}{ }^{-1 / 8} \mathrm{P}_{\text {Wlim }}{ }^{3 / 8}
\end{aligned}
$$

\section{OTHER ASPECTS OF LARGE SIZE}

At constant field for geometrically similar resistive magnets, total coolant pumping power is reduced as magnet size is increased, approximately varying as $P_{\text {pump }} \propto 1 / R$. Stresses in geometrically similar magnets are proportional to the square of magnetic field but are independent of magnet size. Magnet currents vary as the product of linear size and field, as does the plasma current at constant safety factor, q. Peak conductor temperatures at constant field are approximately independent of size. Voltages at constant field are size independent, so correspondingly less insulation is needed in larger magnets. Magnetic flux available from the $\mathrm{OH}$ solenoid is proportional to $\mathrm{R}^{2}$, as is the flux needed to establish plasma current at a constant $q$ value. Electrical and thermal time constants all vary as $\mathrm{R}^{2}$.

One side benefit of large size is that the breeding fusion blanket region becomes a smaller part of the total system volume, and so makes a smaller contribution to COE. A second benefit is that inductively driven pulse durations become extremely long, so cheap inductive plasma current drive can be used with day long or week long pulse durations which would match typical load demand cycles. A third benefit is that ignition would not require "H-mode" plasma confinement since the "L-Mode" confinement regime would be adequate. (At the large sizes envisioned, L-Mode confinement may actually need to be degraded in order to maintain plasma temperature at its optimum value.)

Power plants need to be sufficiently cheap and sufficiently reliable to compete with alternative power sources. They do not additionally need to be small. Although natural gas turbines can be economical in small sizes, other successful technologies require larger unit sizes for economy. As an example, France has retired all electricity producing PWRs (pressurized water nuclear reactors) smaller than $850 \mathrm{Mwe}$, now operates 34 older PWRs at 0.9 Gwe each, now operates 20 newer PWRs at 1.3 Gwe each, has recently (1996) started up a 1.46 Gwe PWR and is now constructing 3 more 1.46 Gwe PWRs. 5 Some of these PWRs use multiple steam turbines for a single reactor, which may increase reliability or may simplify manufacturing. As another example consider hydroelectric plants. China's single "Three Gorges" dam now under construction (and for which millions of people will be relocated) will produce 18 Gwe using multiple water turbines. Many other operating hydroelectric plants around the world have multi-GWe capacities; all are physically gigantic compared to proposed fusion plants, but most are successful due to their cheapness and reliability. Thus the most important issue is not the plant's physical size or its GW power capacity; it is the overall COE, per reliable kw-hr, characteristic of the plant.

\section{RESISTIVE CONDUCTOR ECONOMICS}

Electricity is distributed using resistive conductors (not superconductors). The designer of an electric cable can choose copper or aluminum conductor materials, and can arbitrarily choose the current density, which has no physical restrictions. The initial cable cost per ampere-meter is inversely proportional to the chosen current density, but the recurring future annual cost per ampere-meter is directly proportional to chosen current density. By using an appropriate discounting interest rate, the recurring future annual costs can be converted into an equivalent present 
value comparable to the initial capital cost. The total economic cost of a resistive conductor then includes the present value of all future dissipated power plus the direct expense of the conductor. It thus depends on the designed current density and on the duty factor.

At recent prices, electrical grade copper costs about $\$ 21.5 \mathrm{~K} / \mathrm{m}^{3}$ while electrical grade aluminum costs about $\$ 6.5 \mathrm{~K} / \mathrm{m}^{3}$. Their electrical resistivities at room temperature are $17.2 \mathrm{n} \Omega \mathrm{m}$ and $26 \mathrm{n} \Omega \mathrm{m}$, respectively. Assuming a $100 \%$ duty factor, a $10 \%$ (WACC) discount rate, and costing the dissipated electricity at $\$ 0.05 / \mathrm{kw}-\mathrm{hr}$, the total present value of the economic cost of resistive conductors varies as shown in the following graph

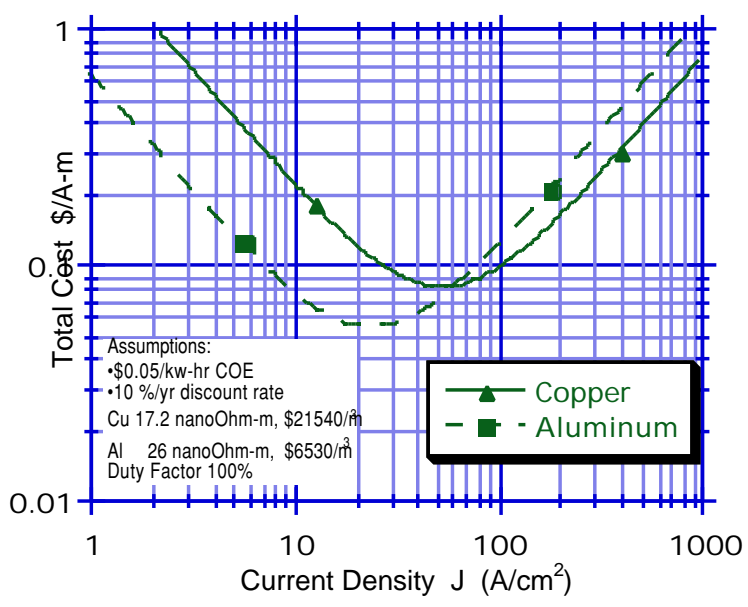

Figure 1: Total Present Value of Resistive Costs

(Present Conductor + Future Dissipation) versus Design Current Density

This graph shows that when there are no volume constraints, the optimum conductor is aluminum, its optimum current density is $25 \mathrm{~A} / \mathrm{cm}^{2}$, and the optimum total present value of initial plus future costs is $\$ 0.053 / \mathrm{amp}$ meter. (At this optimum, cost is split equally between initial and future costs.) The graph further shows that aluminum is more cost effective than copper for current densities less than $60 \mathrm{~A} / \mathrm{cm}^{2}$, but that copper is cheaper for higher current densities. Although the precise parameters of conclusions based on Figure 1 would vary with interest rates and with metals and electricity prices, the qualitative results can be applied to selecting parameters for tokamak fusion reactors using massive resistive electromagnet. In the space-constrained center region, copper is the better conductor choice. For the TF coil outer legs, if there is enough room then aluminum conductor may be cheaper. If the conductors have similar economic cost the aluminum may still have magnet manufacturing advantages due to its lighter weight and its greater ease of shaping.

\section{CRYOGENIC RESISTIVE MAGNETS IN FUSION}

Although cryogenic resistive magnet designs are cost effective alternatives for intermittant pulsed operation of fusion physics experiments, their situation is different for fusion power production. The steady electrical power needed to operate cryogenic refrigerators far exceeds the power dissipated in the magnet and can even exceed the power needed to operate at room temperature without refrigeration. Considering realistic refrigerator efficiencies and costs, liquid nitrogen temperature resistive designs do not appear competitive for MFE reactors. At liquid hydrogen temperatures $\left(14^{\circ} \mathrm{K}-20{ }^{\circ} \mathrm{K}\right)$, resistive magnet designs using high purity aluminum may possibly become competitive.

\section{SCALED ITER PLASMA RESISTIVE EXAMPLES}

Table 2: Massive Resistive Coil MFE Plant Examples

\begin{tabular}{|c|c|c|}
\hline & 2xITER & 5xITER \\
\hline Wall Load $\left(\mathrm{MW} / \mathrm{m}^{2}\right)$ & $5 \mathrm{MW} / \mathrm{m}^{2}$ & $5 \mathrm{MW} / \mathrm{m}^{2}$ \\
\hline Inductive Burn PulseDuration & 1 day & 1 week \\
\hline Confinement & L-mode & L-mode \\
\hline Toroidal Field (@ R) (Tesla) & 8.52 & 5.7 \\
\hline Plasma Current (MA) & 52.8 & 105.0 \\
\hline Major Radius R (m) & 16.28 & 40.70 \\
\hline Minor radius a $(\mathrm{m})$ & 5.6 & 14.0 \\
\hline Elongation & 1.6 & 1.6 \\
\hline Divertor & 1 Null & $1 \mathrm{Null}$ \\
\hline Fusion Power (GW) & 30.00 & 187.50 \\
\hline Total Thermal Power (GWth) & 38.10 & 238.13 \\
\hline Gross Electric Power (GWe) & 12.57 & 78.58 \\
\hline Magnet Power (GWe) & 3.33 & 4.14 \\
\hline Net Power for Sale (GWe) & 9.24 & 74.44 \\
\hline $\begin{array}{l}\text { Annual Value Of Dissipated } \\
\text { MagnetPower } \\
(@ \$ 0.03 / \mathrm{kwhr}, 70 \% \text { capacity })\end{array}$ & $\$ 0.61 \mathrm{~B} / \mathrm{yr}$ & $\$ 0.76 \mathrm{~B} / \mathrm{yr}$ \\
\hline $\begin{array}{l}\text { AnnualRevenues } \\
(@ \$ 0.03 / \mathrm{kwhr}, 70 \% \text { capacity) }\end{array}$ & $\$ 1.7 \mathrm{~B} / \mathrm{yr}$ & $\$ 13.7 \mathrm{~B} / \mathrm{yr}$ \\
\hline CostofMagnet $\mathrm{Al}+\mathrm{Cu}(\$ \mathrm{~B})$ & $\$ 0.46 \mathrm{~B}$ & $\$ 7.50 \mathrm{~B}$ \\
\hline Recirc. Power Fraction, $\mathrm{f}$ & 0.2649 & .0527 \\
\hline COE multiplier $1 /(1-f)$ & 1.360 & 1.056 \\
\hline TFInnerLegCurDens. $\left(\mathrm{A} / \mathrm{cm}^{2}\right)$ & 450 & 94 \\
\hline TFOuterLegCurDens. $\left(\mathrm{A} / \mathrm{cm}^{2}\right)$ & 89 & 33 \\
\hline $\begin{array}{l}\text { Al+Cu Conductor } \\
\text { CapitalCost/NetWattOutput }\end{array}$ & $\$ 0.05$ & $\$ 0.10$ \\
\hline
\end{tabular}

Table 2 gives parameters of two unoptimized MFE tokamak power plants with massively resistive electromagnets. TF magnet parameters were calculated accurately and rough PF estimates added without detailed calculations. Plasma parameters for both examples were scaled from the ITER plasma to achieve $5 \mathrm{MW} / \mathrm{m}^{2}$ wall loading. In both cases a gap of 3 meters (10 feet) was left on all sides between the plasma and the TF for a full tritium breeding blanket, for a vacuum vessel, and an internal PF coil set for steady plasma shaping and control. The TF magnets are demountable with inner legs made of water cooled copper and remaining parts made of water cooled aluminum. Inboard, $70 \%$ of the central volume was filled with TF copper. The aluminum portions of the TF were assigned an expanded cross section. 
The smaller (9.24 Gwe) unit has a cheaper magnet capital cost per net watt output than the larger (74 Gwe) unit, but has a higher recirculating power fraction and COE multiplier which affect "balance-or-plant" costs. Overall economic optimization of unit size thus depends on cost details of other systems, and has not been attempted.

The bottom line of Table 2 should be compared to the entries in Table 1, but with careful interpretation. Since there are no MFE fusion power plant designs comparable to ITER in engineering and costing detail, the ITER experiment design provides useful information against which massive resistive magnet design concepts can be considered. Roughly half of the ITER experiment's capital covers superconducting magnets, their cryogenic refrigeration, and the electrical protection systems accommodating their quench events. But since ITER's superconducting magnets are not large enough for a full tritium breeding blanket surrounding the plasma, the superconducting magnets in an ITER-like MFE reactor would be even larger than ITER's. A MFE reactor's capital cost would also include other elements not in the ITER experiment such as the blanket, tritium extraction, high temperature heat extraction, rotating turbines, electrical generators, cooling towers.

Figure 2 shows an elevation view of the larger MFE unit's TF magnet. Its height would match a 33 story office building. Although this is very large compared with more conventional MFE power plant designs, it is well within the size range of structures which humans have constructed.

\section{Resistive 5X ITER Concept .}

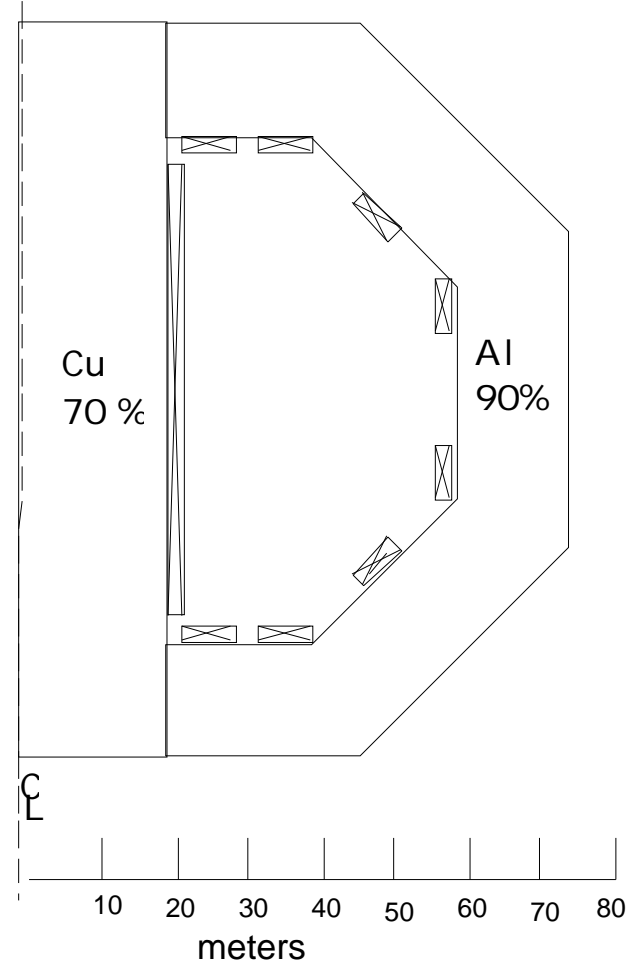

Figure 2: $5 \mathrm{x}$ ITER Elevation View

\section{POSSIBLE PROBLEMS AND DIFFICULTIES}

The Greenwald density limit for ohmically heated tokamaks is a possible "show-stopper" problem for this concept, since for $\mathrm{OH}$ plasmas it scales in proportion to $\mathrm{B} / \mathrm{R}$ which would reduce $\beta$ for larger sizes. However, theories hypothesize this density limit arises as an instability when plasma heating is insufficient to power impurity radiation at the plasma's edge, resulting in a shrinking current channel which leads to plasma disruption. They predict that higher heating power will permit higher density. Experiments have confirmed that the density limit does increase with non-ohmic heating power. J.Wesson has conjectured that a reactor's density limit will increase in proportion to the square root of fusion power, in which case it will not limit an ignited DT plasma. ${ }^{6}$ (We will hope that this conjecture is true.)

At the proposed large plasma size and current, plasma disruptions would be impressive. It becomes even more important than with conventional size tokamaks to learn how to avoid, or at least survive, plasma disruptions.

The very large plant power unit size will require some special premium in order to be economically attractive. It must have a cheaper COE than alternatives. And it must either be quite reliable or have a significantly cheaper COE.

\section{OBSERVATIONS AND CONCLUSIONS}

For this fusion concept, it is not necessary to do much more scientific or engineering research to develop economical fusion reactors. It is not necessary to research enhanced plasma confinement, bootstrap current, profile control, or superconducting magnet technology. L-mode ignition is assured in this concept, but DT burn dynamics need to be experimentally investigated and methods of deliberately degrading L-mode confinement need to be tested.

If the COE can be cheap enough (i.e., $<\$ 0.01 / \mathrm{kw}-\mathrm{hr}$ ), there would be an immediate role for massively large tokamak plants in electrolyzing seawater to make hydrogen fuel, thus replacing an oil field or a coal mine. For this application large unit size is not an impediment. Hydrogen production would also be forgiving of periodic interruptions to reset an $\mathrm{OH}$ solenoid, or rare interruptions following a disruption.

\section{REFERENCES}

1. L. Bromberg, D. Cohn, D. Jassby, "Commercial Tokamak Reactors with Resistive Toroidal Field Magnets", FUSION TECHNOLOGY 6 597-604 (1984)

2. L. Bromberg, D.Cohn, J. Williams, "A Modular Commercial Reactor with Day Long Pulses", J. Fusion Energy 3 63-66 (1983)

3. J.Wesson,Tokamaks, $2^{\text {nd }}$ Ed,ClarendonPress, Oxford,1997

4. G. Miley et al,"Slides\&NotesSPECIAL MINICOURSE ON FUSION BLANKET TECHNOLOGY", 30September1995, Fusion Studies Lab, U.of IL

5. Internet Address http://www.info-franceusa.org/america/embassy/nucleafn.htm

6. J. Wesson et al, "DISRUPTIONS IN JET", NUCLEAR FUSION 29 NO. 4, p641-666, (1989) 